\title{
A higher ratio of refined grain to whole grain is associated with a greater likelihood of chronic kidney disease: a population-based study
}

\author{
Mohsen Mazidi ${ }^{1,2 *}$, Niki Katsiki ${ }^{3}$, Dimitri P. Mikhailidis ${ }^{4}$ and Maciej Banach ${ }^{5,6,7}$ \\ ${ }^{1}$ Key State Laboratory of Molecular Developmental Biology, Institute of Genetics and Developmental Biology, Chinese Academy \\ of Sciences, Chaoyang, People's Republic of China \\ ${ }^{2}$ Institute of Genetics and Developmental Biology, International College, University of Chinese Academy of Science (IC-UCAS), \\ Chaoyang, People's Republic of China \\ ${ }^{3}$ Second Propedeutic Department of Internal Medicine, Medical School, Aristotle University of Thessaloniki, Hippokration \\ Hospital, 54642 Thessaloniki, Greece \\ ${ }^{4}$ Department of Clinical Biochemistry, Royal Free Campus, University College London Medical School, University College \\ London, London NW3 2QG, UK \\ ${ }^{5}$ Department of Hypertension, Chair of Nephrology and Hypertension, Medical University of Lodz, Lodz, Poland \\ ${ }^{6}$ Department of Hypertension, Polish Mother's Memorial Hospital Research Institute (PMMHRI), Lodz, Poland \\ ${ }^{7}$ Cardiovascular Research Centre, University of Zielona Gora, Zielona Gora, Poland
}

(Submitted 21 January 2018 - Final revision received 9 August 2018 - Accepted 5 September 2018 - First published online 7 June 2019)

\section{Abstract}

A growing number of studies suggest that diet and renal function are related. However, little is known about the link between both whole grain (WG) and refined grain (RG) consumption and kidney function parameters. Thus, we investigated the association of WG and RG with urinary albumin to creatinine ratio (ACR) and prevalent chronic kidney disease (CKD). Data from participants of the National Health and Nutrition Examination Surveys (NHANES) from 2005 to 2010 were collected. Estimated glomerular filtration rate (eGFR) was calculated by the CKD Epidemiology Collaboration equation. Survey design and sample weights were taken into consideration for statistical analyses. Finally, we included 16325 participants from NHANES, 6.9\% of whom had prevalent CKD. In models adjusted for age, sex, race, fasting blood glucose, blood pressure, adiposity, hypertension and diabetes status, mean eGFR significantly increased across increasing quartiles of WG (Q1: $88.2 v$. Q4: $95.4 \mathrm{ml} / \mathrm{min}$ per $1.73 \mathrm{~m}^{2}, P<0.001$ ), whereas it significantly decreased across increasing quartiles of RG (Q1: $97 \cdot 2 v$. Q4: $88.4 \mathrm{ml} / \mathrm{min}$ per $1.73 \mathrm{~m}^{2}, P<0.001$ ). Furthermore, serum uric acid levels and ACR significantly decreased across quartiles of WG (both $P<0 \cdot 001)$. In multivariable-adjusted logistic regression models, the likelihood of prevalent CKD was $21 \%$ lower in the highest WG quartile compared with the lowest one. In conclusion, our results shed light on the beneficial impact of WG on kidney function and CKD, whereas RG is adversely associated with eGFR.

Key words: Whole grain: Refined grain: Chronic kidney disease: Uric acid: Estimated glomerular filtration rate: Urinary albumin to creatinine ratio

Chronic kidney disease (CKD) is increasingly recognised as a major global public health problem. In 2016, thirty million people or $15 \%$ of US adults are estimated to have $\mathrm{CKD}^{(1)}$. Patients with CKD are at increased risk for CVD, end-stage renal disease (ESRD) and all-cause mortality ${ }^{(1-3)}$. The 2010 US Renal Data System report shows that Medicare spent $\$ 29$ billion in 2009 , or almost $6 \%$ of the annual Medicare budget, for patients with $\mathrm{ESRD}^{(4)}$. Primary prevention of CKD is therefore an important public health priority ${ }^{(5)}$. Inflammation seems to play an important role in the pathogenesis of kidney injury, leading to chronically impaired kidney function ${ }^{(5,6)}$.

It is known that diet plays a critical role in the regulation of the level of inflammatory factors and oxidative stress ${ }^{(7-9)}$ and possibly in renal function. It has been suggested that diets with high load of nutrients such as antioxidant vitamins including vitamin E, C, A, Mg, K, Ca, fibre, PUFA, MUFA and phytochemicals and a lower amount of SFA, trans-fatty acids and simple carbohydrate could have a beneficial impact on renal

Abbreviations: ACR, albumin to creatinine ratio; CKD, chronic kidney disease; CRP, C-reactive protein; DM, diabetes mellitus; eGFR, estimated glomerular filtration rate; HTN, hypertension; MESA, Multi-Ethnic Study of Atherosclerosis; NHANES, National Health and Nutrition Examination Survey; RG, refined grain; WG, whole grain.

* Corresponding author: M. Mazidi, email moshen@genetics.ac.cn 
function and decrease the likelihood of $\operatorname{CKD}^{(10,11)}$. Some nutrients, such as fibre, and several vitamins are recognised as anti-inflammatory nutrients ${ }^{(12)}$, and some of them including SFA or sugar are known to have a pro-inflammatory action ${ }^{(13)}$. The first category is related to favourable renal function, lower risk of albuminuria and slower decline in kidney function ${ }^{(12,14,15)}$, while the second group of nutrients has been linked with worsening of kidney function ${ }^{(16)}$.

However, there is a limited number of controversial evidence on the association between whole grain (WG) or refined grain (RG) and kidney function ${ }^{(17-19)}$. The Multi-Ethnic Study of Atherosclerosis (MESA) showed that higher consumption of WG was associated with lower albumin to creatinine ratio $(\mathrm{ACR})^{(20)}$. In contrast, the Framingham Heart Study found no association between consumption of WG, microalbuminuria and low estimated glomerular filtration rate (eGFR) ${ }^{(19)}$. Very recently, results from the population-based longitudinal Doetinchem study ( $n$ 3787, 26-65 years) reported no link between WG intake, eGFR and $\mathrm{ACR}^{(18)}$.

The main objectives in the management of CKD include: reduction of the unfavourable symptoms of uraemia, delaying the need for renal replacement therapy and improving the quality of life ${ }^{(21)}$. The dietary limitations for CKD subjects make it difficult to ensure adequate micronutrient content in their diet. Furthermore, they may suffer from impaired intestinal absorption of mineral and vitamins ${ }^{(22)}$.

The aim of this study was to investigate the association of WG and RG with kidney function (assessed by eGFR) and prevalent CKD in adult Americans. Second, we evaluated the ratio of WG:RG in the diet in relation to CKD prevalence. Furthermore, we examined whether possible associations were moderated by adiposity factors. We hypothesised that individuals with a higher intake of WG would have a more favourable eGFR and a lower likelihood for CKD and vice versa for RG.

\section{Methods}

\section{Population}

We used data from the National Health and Nutrition Examination Survey (NHANES), which is described in detail elsewhere ${ }^{(23)}$. In brief, these are periodic cross-sectional surveys conducted by the US National Center for Health Statistics (NCHS), and during which home visits are conducted to administer questionnaires and collect data on demographics, diet and other health behaviours. NHANES applies complex multistage probability sampling procedure to ensure selection of participants from various geographical locations and adequate racial/ethnic representation ${ }^{(23)}$. Informed consent was obtained from all participants and the NCHS Research Ethics Review Board approved the protocol. Poverty to income index was measured by using the Department of Health and Human Services' poverty guidelines. Smoking status was self-reported and participants classified as current smoker or not. The metabolic equivalent of task (MET) was used to measure the intensity level of physical activity and indicated the rate of energy consumption for a specific activity. A MET is defined as $4.184 \mathrm{~kJ} / \mathrm{kg}$ per $\mathrm{h}(1 \mathrm{kcal} / \mathrm{kg}$ per $\mathrm{h}$ ) that is roughly equal to the energy cost of being at rest. Physical activity was categorised into three intensity levels based on the MET score: light, moderate and vigorous ${ }^{(24)}$.

The methods for the biochemical analyses are described in the NHANES Laboratory/Medical Technologists Procedures Manual $^{(25-28)}$. A blood specimen was drawn from an antecubital vein according to a standardised protocol. Fasting glucose was measured in the plasma by a hexokinase method using a Roche/Hitachi 911 Analyzer. The DxC800 modular chemistry side uses the Jaffe rate method (kinetic alkaline picrate) to determine the concentration of creatinine in serum. The creatinine calibration is traceable to an isotope dilution MS reference method ${ }^{(29)}$. Urinary creatinine (assessed by the Jaffe rate reaction) and urinary albumin (assessed by a solid-phase fluorescent immunoassay from a random urine sample) $)^{(30)}$ were used to calculate ACR. The CKD Epidemiology Collaboration equation was used to calculate eGFR (in $\mathrm{ml} / \mathrm{min}$ per $1.73 \mathrm{~m}^{2}$ ). Prevalent CKD was defined as an eGFR $<60 \mathrm{ml} / \mathrm{min}$ per $1.73 \mathrm{~m}^{2(30)}$. The National Cholesterol Education Program's Adult Treatment Panel III report criteria were used to define the metabolic syndrome (MetS) $)^{(31)}$, based on the presence of three or more of the following five criteria: (1) waist circumference $\geq 102 \mathrm{~cm}$ for men or $\geq 88 \mathrm{~cm}$ for women; (2) TAG $\geq 150 \mathrm{mg} / \mathrm{dl}$ ( $1.7 \mathrm{mmol} / \mathrm{l})$; (3) HDL-cholesterol $<40 \mathrm{mg} / \mathrm{dl}(1.0 \mathrm{mmol} / \mathrm{l})$ for men or $<50 \mathrm{mg} / \mathrm{dl}$ ( $1.3 \mathrm{mmol} / \mathrm{l})$ for women; (4) systolic blood pressure $\geq 130$ or diastolic blood pressure $\geq 85 \mathrm{mmHg}$ and (5) fasting blood glucose $\geq 100 \mathrm{mg} / \mathrm{dl}(5.5 \mathrm{mmol} / \mathrm{l})$.

For the present analysis, three survey cycles (i.e. 2005-2006, 2007-2008 and 2009-2010) were combined to produce estimates with greater precision and smaller sampling error. The analytical sample was limited to adults aged $\geq 18$ years. After excluding pregnant and lactating women ( $n$ 102) as well as those with missing information regarding the variables of interest ( $n$ 2131), the final analytical sample included 16325 respondents from the NHANES 2005-2010.

\section{Whole grain and refined grain consumption}

Dietary intake was assessed via $24 \mathrm{~h}$ recall obtained by a trained interviewer during the mobile examination centre visit, with the use of a computer-assisted dietary interview system with standardised probes, that is, the US Department of Agriculture Automated Multiple-Pass Method (AMPM) ${ }^{(32,33)}$. Briefly, the type and quantity of all foods and beverages consumed in a single $24 \mathrm{~h}$ period before the dietary interview (from midnight to midnight) were collected with the use of the AMPM. The AMPM is designed to enhance complete and accurate data collection while reducing respondent burden ${ }^{(33,34)}$. Detailed descriptions of the dietary interview methods are provided in the NHANES Dietary Interviewer's Training Manual ${ }^{(35)}$. The MyPyramid Equivalents Database for US Department of Agriculture (USDA) Survey Food Codes was used to calculate WG and RG intake ${ }^{(35)}$. The MyPyramid Equivalents Database is currently the only database available that provides quantified measures of WG foods with separate tables based on the old and new (without bran) definitions for WG. My Pyramid Equivalents food data files include the number of servings. Examples of WG food servings within the database include one 
slice of $100 \%$ WG bread, one cup of $100 \%$ WG cereal, or onehalf cup of $100 \%$ WG hot cereal, cooked pasta, rice, or other grain such as bulgur, oatmeal and whole cornmeal. The USDA Community Nutrition Research Group FoodLink staff, in consultation with the Agricultural Research Service's Nutrient Data Laboratory, has classified all grain ingredients used in the Food and Nutrient Database for Dietary Studies and the Continuing Survey of Food Intake by Individuals (CSFII) recipe databases as WG or $\mathrm{RG}^{(33)}$. The total number of ounce equivalents of grains $/ 100 \mathrm{~g}$ has been determined for each food. The total number of ounce equivalents was divided into WG and RG equivalents based on the proportion of the grain ingredients in the food that were WG or non-WG. For example, cracked wheat bread contains white wheat flour and bran, which are RG, and whole-wheat flour, which is a WG. The white wheat flour and bran contribute $68 \%$ to the total weight of the grain ingredients and the whole-wheat flour contributes $32 \%$. Thus, of the four ounce equivalents $/ 100 \mathrm{~g}$ of cracked wheat bread, 2.7 are $\mathrm{RG}$ and 1.3 are $\mathrm{WG}^{(33)}$.

\section{Statistical analysis}

Data analyses followed the Centers for Disease Control and Prevention guidelines for analysis of complex NHANES data, accounting for the masked variance and using the recommended weighting methodology ${ }^{(36)}$, implemented with the use of SPSS ${ }^{\circledR}$ complex sample module version 22.0 (IBM Corp.). We used means and standard errors of the mean for continuous (ANOVA) measures and percentages for categorical variables $\left(\chi^{2}\right)$. The Kolmogorov-Smirnov test was used to evaluate the normality of data. Adjusted mean levels of kidney function markers (serum creatinine, ACR, eGFR, serum uric acid) across WG and RG quartiles were conducted using ANCOVA. These models were adjusted for age, sex, race, poverty:income ratio, fasting blood glucose, systolic and diastolic blood pressure, BMI $\left(\mathrm{kg} / \mathrm{m}^{2}\right)$, diabetes mellitus (DM) (self-reported history of DM or fasting plasma glucose $\geq 126 \mathrm{mg} / \mathrm{dl}$ $(7 \mathrm{mmol} / \mathrm{l})$ ) and hypertension (HTN), diagnosed in individuals with systolic blood pressure $\geq 140 \mathrm{mmHg}$, a diastolic blood pressure $\geq 90 \mathrm{mmHg}$ or in those on antihypertensive drugs ${ }^{(37)}$. Log transformations were performed for those with not normal distribution. Logistic regressions models with three different levels of adjustments (model 1: age, sex, race and poverty:income ratio, model 2: age, sex, race, poverty:income ratio, fasting blood glucose, systolic and diastolic blood pressure and HTN, model 3: age, sex, race, poverty:income ratio, fasting blood glucose, systolic and diastolic blood pressure, HTN, TAG and HDL, DM, BMI and C-reactive protein (CRP)) were then used to derive the OR and $95 \%$ $\mathrm{CI}$ for the association with prevalent CKD across WG, RG and ratio of WG:RG quartiles, always using the lowest quartile as reference. Multi-collinearity for the multiple linear regressions was assessed with variance inflation factors (VIF) at each step $^{(38)}$. Multicollinearity was considered high when the VIF was $>10^{(38)}$.

The SPSS macro for moderation model by Preacher \& Hayes $^{(39)}$ was used to investigate the effects of adiposity (assessed by BMI) on the associations of WG and RG with eGFR. The application of this macro allowed simultaneous estimation of the moderation impact of adiposity, while adjusting for relevant confounders. The approach also allowed the visualisation of the impact of each standard deviation change in the potential moderator on the relationship between independent and dependent variables. We tested for the presence of an effect of the adiposityadjusted model (age, sex, race, fasting blood glucose, systolic and diastolic blood pressure, BMI, DM and HTN). All tests were two-sided, and $P<0.05$ characterised significant results.

\section{Results}

A total of 16325 participants met the criteria for inclusion in the current analysis; $6.9 \%$ had prevalent CKD. The characteristics of participants overall and by status of prevalent CKD are summarised in Table 1 . Overall, $51.3 \%$ of the participants were women, with no significant sex difference between those with and those without CKD $(P=0 \cdot 352)$. Compared with those without CKD, participants with CKD were more likely to be non-Hispanic Whites ( $82.7 v$. 68.6\%), and less likely MexicanAmericans (2.5 v. 8.8\%), non-Hispanic Black (8.3v. 11.2\%) and other Hispanic $(3.1$ v. $5.3 \%) ; P<0.001$ shows differences in the distribution of ethnicity by status for CKD. Mean age was $45 \cdot 8$ years overall; participants with CKD were older than those without $(69.2 v$. 44.6 years, $P<0.001)$. With regard to other characteristics, patients with CKD had a higher BMI $(P<0 \cdot 001)$ and higher serum concentrations of CRP, TAG, fasting and $2 \mathrm{~h}$ glucose, insulin and $\mathrm{HbA}_{1 \mathrm{c}}(P<0.001$ for all comparisons). They were also more likely to have a high prevalence of the MetS, DM and HTN $(P<0.001$ for all comparisons, Table 1$)$.

Adjusted (age, sex, race, fasting blood glucose, systolic and diastolic blood pressure, BMI, DM and HTN) mean levels of kidney function markers by quartiles of WG and RG are shown in Table 2. Across increasing WG quartiles, mean serum uric acid decreased from 6.01 to $5.02 \mathrm{mg} / \mathrm{dl}(357$ to $299 \mu \mathrm{mol} / \mathrm{l})(P<0 \cdot 001)$, urine albumin increased from 2.32 to $1.98 \mathrm{mg} / 1(P<0 \cdot 001)$, while eGFR increased from 88.2 to $95.4 \mathrm{ml} / \mathrm{min}$ per $1.73 \mathrm{~m}^{2}(P<0.001)$. Log ACR also decreased from $2 \cdot 19$ to $1.99(P=0.012)$. With regard to the consumption of RG, we have found that as the quartiles of RG increased, mean serum uric acid increased from 4.99 to $5.78 \mathrm{mg} / \mathrm{dl}(297$ to $344 \mu \mathrm{mol} / \mathrm{l})(P<0.001), \log$ ACR increased from 2.01 to $2.22 \mathrm{mg} / \mathrm{dl}(P<0 \cdot 001)$, whereas eGFR decreased from 97.2 to $88.4 \mathrm{ml} / \mathrm{min}$ per $1.73 \mathrm{~m}^{2}$ ( $P<0.001$, Table 2$)$.

As shown in Table 3, three different models were used with a wide range of potential confounders to evaluate the odds of CKD across the quarters of WG and RG, as well as the ratio of WG:RG. In the model adjusted for age, sex, race and poverty: income ratio compared with the lowest quartile of the WG, the OR for CKD was 0.52 (95\% CI 0.41-0.63) for the top quartiles $(P<0.001$ for trend). In the same model, subjects in the top quarter (Q4) of WG:RG ratio had a lower chance for CKD compared with the first quarter (OR: $0.48,95 \%$ CI $0.34,0.59$, Table 3), while in same model as the quarters of RG increased, the likelihood of CKD was also increasing in a step-wise manner ((Q2 = OR: 1·32, $95 \%$ CI 1·06, 1·46), (Q3 = OR: 1·56, $95 \% \mathrm{CI}$ $1 \cdot 11,1.69)$ and (Q4=OR: $1.78,95 \%$ CI $1.23,1.89, P<0.001)$, Table 3). In the expanded models with further adjustment for age, sex, race, poverty:income ratio, fasting blood glucose, systolic and diastolic blood pressure, HTN, DM, TAG, HDL and $\mathrm{CRP}$, similar associations were observed, that is, as the quarters 
Table 1. Demographic characters of subjects based on chronic kidney disease (CKD) status (Mean values with their standard errors; percentages; mean values and $95 \%$ confidence intervals)

\begin{tabular}{|c|c|c|c|c|c|c|c|}
\hline \multirow[b]{2}{*}{ Characteristics } & \multicolumn{2}{|c|}{ Overall } & \multicolumn{2}{|c|}{ With CKD } & \multicolumn{2}{|c|}{ Without CKD } & \multirow[b]{2}{*}{$P$} \\
\hline & Mean & SEM & Mean & SEM & Mean & SEM & \\
\hline Sex & \multirow{2}{*}{\multicolumn{2}{|c|}{48.7}} & & & & & $<0.001$ \\
\hline Men (\%) & & & \multicolumn{2}{|c|}{37.7} & \multicolumn{2}{|c|}{$49 \cdot 2$} & \\
\hline Women (\%) & \multicolumn{2}{|c|}{$51 \cdot 3$} & \multicolumn{2}{|c|}{$62 \cdot 3$} & \multicolumn{2}{|c|}{$50 \cdot 8$} & \\
\hline Age (years) & & & & & & & $<0.001$ \\
\hline Mean & \multicolumn{2}{|c|}{$45 \cdot 8$} & \multicolumn{2}{|c|}{69.2} & \multicolumn{2}{|c|}{44.6} & \\
\hline $95 \% \mathrm{Cl}$ & \multicolumn{2}{|c|}{0.1} & \multicolumn{2}{|c|}{0.3} & \multicolumn{2}{|c|}{0.2} & \\
\hline Race/ethnicity & & & & & & & $<0.001$ \\
\hline White (non-Hispanic) (\%) & \multicolumn{2}{|c|}{$68 \cdot 4$} & \multicolumn{2}{|c|}{82.7} & \multicolumn{2}{|c|}{68.6} & \\
\hline Non-Hispanic Black (\%) & \multicolumn{2}{|c|}{11.5} & \multicolumn{2}{|c|}{8.3} & \multicolumn{2}{|c|}{11.2} & \\
\hline Mexican-American (\%) & \multicolumn{2}{|c|}{8.1} & \multicolumn{2}{|c|}{$2 \cdot 5$} & \multicolumn{2}{|c|}{8.8} & \\
\hline Other Hispanic (\%) & \multicolumn{2}{|c|}{$5 \cdot 2$} & \multicolumn{2}{|c|}{3.1} & \multicolumn{2}{|c|}{$5 \cdot 3$} & \\
\hline BMI $\left(\mathrm{kg} / \mathrm{m}^{2}\right)$ & $28 \cdot 3$ & $0 \cdot 1$ & $29 \cdot 1$ & 0.1 & 28.5 & 0.1 & $<0.001$ \\
\hline Serum TAG $(\mathrm{mg} / \mathrm{dl})^{*}$ & $155 \cdot 3$ & 3.0 & $179 \cdot 2$ & 3.9 & 151.9 & $2 \cdot 3$ & $<0.001$ \\
\hline Serum total cholesterol $(\mathrm{mg} / \mathrm{dl})^{\star}$ & $196 \cdot 1$ & 0.7 & $192 \cdot 9$ & 1.0 & $196 \cdot 3$ & 0.8 & 0.125 \\
\hline Serum HDL $(\mathrm{mg} / \mathrm{dll})^{\star}$ & $53 \cdot 2$ & 0.5 & $53 \cdot 2$ & 0.4 & 53.2 & 0.2 & 0.362 \\
\hline Serum CRP $(\mathrm{mg} / \mathrm{dl})^{*}$ & 0.30 & 0.03 & 0.54 & 0.02 & 0.32 & 0.01 & $<0.001$ \\
\hline Systolic blood pressure $(\mathrm{mmHg})$ & $121 \cdot 9$ & 0.4 & $133 \cdot 2$ & 0.5 & $121 \cdot 0$ & 0.2 & $<0.001$ \\
\hline Diastolic blood pressure $(\mathrm{mmHg})$ & $70 \cdot 2$ & 0.4 & 69.9 & 0.2 & 65.4 & 0.2 & $<0.001$ \\
\hline Fasting blood glucose $(\mathrm{mg} / \mathrm{dll})^{\star}$ & 98.5 & 0.2 & $113 \cdot 0$ & 0.8 & $97 \cdot 3$ & 0.3 & $<0.001$ \\
\hline Plasma insulin $(\mu \mathrm{U} / \mathrm{ml})$ & $13 \cdot 0$ & 0.1 & 14.6 & 0.1 & $12 \cdot 7$ & 0.1 & $<0.001$ \\
\hline $\mathrm{HbA}_{1 \mathrm{c}}(\%)$ & 5.5 & 0.01 & $6 \cdot 0$ & 0.01 & 5.6 & 0.01 & $<0.001$ \\
\hline Hypertension (\%) & \multicolumn{2}{|c|}{$15 \cdot 3$} & & & & & $<0.001$ \\
\hline Diabetes (\%) & & & & & & & $<0.001$ \\
\hline Metabolic syndrome (\%) & & & & & & & $<0.001$ \\
\hline Energy intake (kJ) & $8602 \cdot 30$ & 28.45 & 7791.44 & 44.35 & $9058 \cdot 36$ & $30 \cdot 54$ & $<0.001$ \\
\hline Energy intake (kcal) & $2056 \cdot 0$ & $6 \cdot 8$ & $1862 \cdot 2$ & $10 \cdot 6$ & $2165 \cdot 0$ & $7 \cdot 3$ & $<0.001$ \\
\hline Total fat intake $(\mathrm{g})$ & 78.0 & 0.3 & $74 \cdot 2$ & 0.9 & 80.5 & 0.3 & $<0.001$ \\
\hline Saturate fat $(\mathrm{g})$ & $25 \cdot 7$ & 0.1 & 24.9 & 0.2 & 26.9 & 0.1 & $<0.001$ \\
\hline Fibre (g) & $16 \cdot 2$ & 0.1 & 13.9 & 0.2 & $16 \cdot 5$ & 0.1 & $<0.001$ \\
\hline
\end{tabular}

CRP, C-reactive protein; $\mathrm{HbA}_{1 \mathrm{c}}$, glycated $\mathrm{Hb}$.

* To convert TAG from mg/dl to mmol/l, multiply by 0.0113 . To convert cholesterol from $\mathrm{mg} / \mathrm{dl}$ to mmol/l, multiply by 0.0259 . To convert CRP from mg/dl to mg/l, multiply by 10 . To convert glucose from $\mathrm{mg} / \mathrm{dl}$ to $\mathrm{mmol} / \mathrm{l}$, multiply by 0.0555 .

of WG and ratio of WG:RG increased, the likelihood for CKD was decreasing $(P<0.001$ for both, Table 3$)$, whereas the second quarter of RG was no longer significant after adjustment for age, sex, race, poverty:income ratio, fasting blood glucose, systolic and diastolic blood pressure, HTN, DM, TAG, HDL and CRP ((Q2= OR: 1·00, $95 \%$ CI 0.98, 1.01), (Q3= OR: $1 \cdot 02,95 \%$ CI 1.01, 1.03) and (Q4=OR: 1.07, $95 \%$ CI 1.05, 1.09, $P<0.001$ for trend, Table 3 ).

The results of the moderation analysis to investigate the role of adiposity (assessed by BMI) in the association between WG and RG intake with eGFR are shown in Fig. 1(a) and (b). In the model adjusted for age, sex, race, poverty:income ratio, alcohol intake, energy intake, smoking, physical activity, fasting blood glucose, systolic and diastolic blood pressure, HTN, DM, TAG, HDL and CRP, BMI moderated the link between WG and eGFR, for example, when WG consumption changed from low to high, eGFR in the low BMI category $\left(21.3 \mathrm{~kg} / \mathrm{m}^{2}\right)$ changed from 96.2 to $107.2 \mathrm{ml} / \mathrm{min}$ per $1.73 \mathrm{~m}^{2}$ (an increase of $11 \mathrm{ml} / \mathrm{min}$ per $\left.1.73 \mathrm{~m}^{2}\right)$, whereas in the high BMI category $\left(36.3 \mathrm{~kg} / \mathrm{m}^{2}\right)$ it changed from 77.4 to $83.2 \mathrm{ml} / \mathrm{min}$ per $1.73 \mathrm{~m}^{2}$ (an increase of $5.8 \mathrm{ml} / \mathrm{min}$ per $1.73 \mathrm{~m}^{2}$ ), thus suggesting that obesity strongly modulates the impact of WG on eGFR, particularly in the group of low BMI (Fig. 1(a)). With regard to RG consumption, in the low BMI category, eGFR varied from 99.6 to $93.2 \mathrm{ml} / \mathrm{min}$ per $1.73 \mathrm{~m}^{2}\left(6.4 \mathrm{ml} / \mathrm{min}\right.$ per $1.73 \mathrm{~m}^{2}$ increase $)$, whereas in the high BMI category, it changed from 82.1 to $71.3 \mathrm{ml} / \mathrm{min}$ per $1.73 \mathrm{~m}^{2}$ $\left(10.8 \mathrm{ml} / \mathrm{min}\right.$ per $1.73 \mathrm{~m}^{2}$ increase), indicating that in contrast to WG intake, a higher BMI had a greater impact on the link of RG with eGFR (Fig. 1(b)).

\section{Discussion}

To our knowledge, the present study is the largest study that examined the association of WG, RG and WG:RG ratio with CKD prevalence and renal function markers in a representative sample of US adults. We found that participants with a higher intake of WG had better markers of renal function, while RG adversely affected these markers. Furthermore, participants with a greater intake of WG or WG:RG ratio had a lower risk for CKD, whereas subjects with a higher RG consumption had a greater likelihood of CKD. Adiposity, assessed by BMI, significantly moderated the link between WG and RG with eGFR. Our findings were robust even after adjustment for a wide range of confounders.

Our results are in accordance with other studies. The prospective observational Nurses' Health Study reported that women in the top quartile of the Dietary Approaches to Stop Hypertension (DASH) score (highly loaded with WG sources) had a decreased risk of rapid eGFR decline (OR: 0.55; $95 \% \mathrm{CI}$ $0 \cdot 38,0 \cdot 80)$ compared with the lowest quartile ${ }^{(33)}$. Furthermore, the highest quartile of the Western pattern score (rich in with 
$\mathrm{RG}^{(8)}$ ) was associated with a high risk of microalbuminuria (OR: 2.17; $95 \%$ CI 1.18, 3.66) and rapid eGFR decline $\geq 3 \mathrm{ml} / \mathrm{min}$ per $1.73 \mathrm{~m}^{2}$ per year (OR: $1.77 ; 95 \%$ CI $\left.1.03,3.03\right)^{(40)}$. The MESA ( $n$ 5000) demonstrated that a dietary pattern rich in WG was associated with a lower ACR (20\% lower ACR across quintiles, $P=0.004)$, whereas a dietary pattern which is rich in animal food was directly associated with an $11 \%$ higher ACR across quintiles $(P=0.03)^{(20)}$. Similarly, the Healthy Eating Index ${ }^{(39)}$ was associated with a lower risk of albuminuria and eGFR decline in DM patients ${ }^{(41,42)}$. The Northern Manhattan Study (NOMAS) reported that a high vegetable intake (the main source of WG) was related to a significantly lower risk of incident eGFR $<60 \mathrm{ml} / \mathrm{min}$ per $1.73 \mathrm{~m}^{2(43)}$. However, results from the Doetinchem Study ( $n$ 3787, 26-65 years) reported no link between WG intake, eGFR and mean $\mathrm{ACR}^{(18)}$, indicating that further research is needed to replicate these findings in other large populations. Discrepancies in these findings may be due to differences in study design. In this context, participants of the MESA study included older adults of mixed origin, whereas in the Doetinchem Study there were relatively young, white individuals with well-preserved renal function.

In this context, findings from the prospective Framingham Heart Study and NOMAS showed that a higher diet quality ${ }^{(19,44)}$, a better adherence to a Mediterranean ${ }^{(43)}$ or a DASH diets, but not prudent $\operatorname{diet}^{(45)}$ was associated with a reduced incidence of low eGFR or risk of rapid eGFR decline. As a higher intake of WG and limited consumption of RG are highly recommended in these dietary patterns, it is expected that these individual foods are associated with renal function too, which is in accordance with our results.

Poor diet could lead to several tissue-specific and systemic metabolic disturbances that could enhance renal dysfunction $^{(46,47)}$. For example, diets with high load of WG are inversely linked with inflammatory factors, whereas a diet with high amount of total fats and processed meats is positively related with inflammatory factors ${ }^{(48)}$. Of note, a link between inflammation and unfavourable renal profile was proposed ${ }^{(49)}$. Taken together with our results, decreasing the level of inflammation (increasing load of WG and ratio of WG:RG) should be a reasonable target to control and prevent the developments of CKD.

There is a biological plausibility that WG may protect against declining kidney function. Possible mechanism might be associated with the endothelial dysfunction, which has been associated with both CKD and cardiovascular risk, and has been favourably affected by a WG intake ${ }^{(50)}$. Moreover, some studies reported a beneficial impact of WG intake on inflammation ${ }^{(50-52)}$, which is closely linked to CKD (as explained before). Lipid profile and blood pressure have also been favourably affected by WG consumption, which may also translate into beneficial kidney effects ${ }^{(53,54)}$. Of note, statins that exert anti-inflammatory and hypolipidaemic effects have also been shown to improve kidney function $^{(55-58)}$. It has been reported that WG are a rich source of fibre, minerals (Mg, K, P, Se, Mn, Zn and Fe), vitamins (particularly high in vitamins $\mathrm{B}$ and $\mathrm{E}$ ), phenolic compounds, phytooestrogens (lignans) and related antioxidants ${ }^{(59)}$. These compounds have been found to favourably affect markers of inflammation and glucose homoeostasis ${ }^{(60,61)}$. Moreover, WG intake may produce SCFA in the large intestine which have beneficial effects on insulin/glucose management ${ }^{(62)}$. 
Table 3. Adjusted logistic regression to examine the association between quartiles for whole grain (WG), refined grain (RG), WG:RG and the risk of chronic kidney disease (CKD)*

(Odds ratios and lower bound-upper bounds)

\begin{tabular}{|c|c|c|c|c|c|c|}
\hline \multirow[b]{3}{*}{ Variables } & \multicolumn{6}{|c|}{ Likelihood of CKD with different models } \\
\hline & \multicolumn{2}{|c|}{$\begin{array}{l}\text { Age, sex, race, poverty:income } \\
\text { ratio }\end{array}$} & \multicolumn{2}{|c|}{$\begin{array}{c}\text { Age, sex, race, poverty:income ratio, alcohol intake, } \\
\text { energy intake, smoking, physical activity, fasting } \\
\text { blood glucose, systolic and diastolic blood } \\
\text { pressure, HTN, DM }\end{array}$} & \multicolumn{2}{|c|}{$\begin{array}{l}\text { Age, sex, race, poverty:income ratio, alcohol intake, } \\
\text { energy intake, smoking, physical activity, fasting } \\
\text { blood glucose, systolic and diastolic blood } \\
\text { pressure, HTN, DM, TAG and HDL, CRP }\end{array}$} \\
\hline & OR & Lower bound-upper bound & OR & Lower bound-upper bound & OR & Lower bound-upper bound \\
\hline WG (Q2) & 0.77 & $0.66-0.86$ & 0.84 & $0.71-0.91$ & 0.95 & $0.78-0.98$ \\
\hline WG (Q3) & 0.72 & $0.62-0.82$ & 0.79 & $0.67-0.87$ & 0.87 & $0.72-0.92$ \\
\hline WG (Q4) & 0.52 & $0.41-0.63$ & 0.66 & $0.52-0.74$ & 0.78 & $0.68-0.87$ \\
\hline RG (Q2) & 1.32 & $1.06-1.46$ & 1.04 & $1.02-1.09$ & 1.00 & $0.98-1.01$ \\
\hline RG (Q3) & 1.56 & $1.11-1.69$ & 1.12 & $1.08-1.23$ & 1.02 & $1.01-1.03$ \\
\hline RG (Q4) & 1.78 & $1.23-1.89$ & 1.22 & $1.11-1.31$ & 1.07 & $1.05-1.09$ \\
\hline WG:RG (Q2) & 0.63 & $0.52-0.75$ & 0.64 & $0.54-0.79$ & 0.71 & $0.63-0.82$ \\
\hline WG:RG (Q3) & 0.66 & $0.55-0.81$ & 0.62 & $0.52-0.76$ & 0.68 & $0.59-0.78$ \\
\hline WG:RG (Q4) & 0.48 & $0.34-0.59$ & 0.51 & $0.42-0.62$ & 0.55 & $0.42-0.66$ \\
\hline
\end{tabular}

HTN, hypertension; DM, diabetes mellitus; CRP, C-reactive protein; Q2, second quarter; Q3, third quarter; Q4, fourth quarter. * The first quarter was always used as reference.
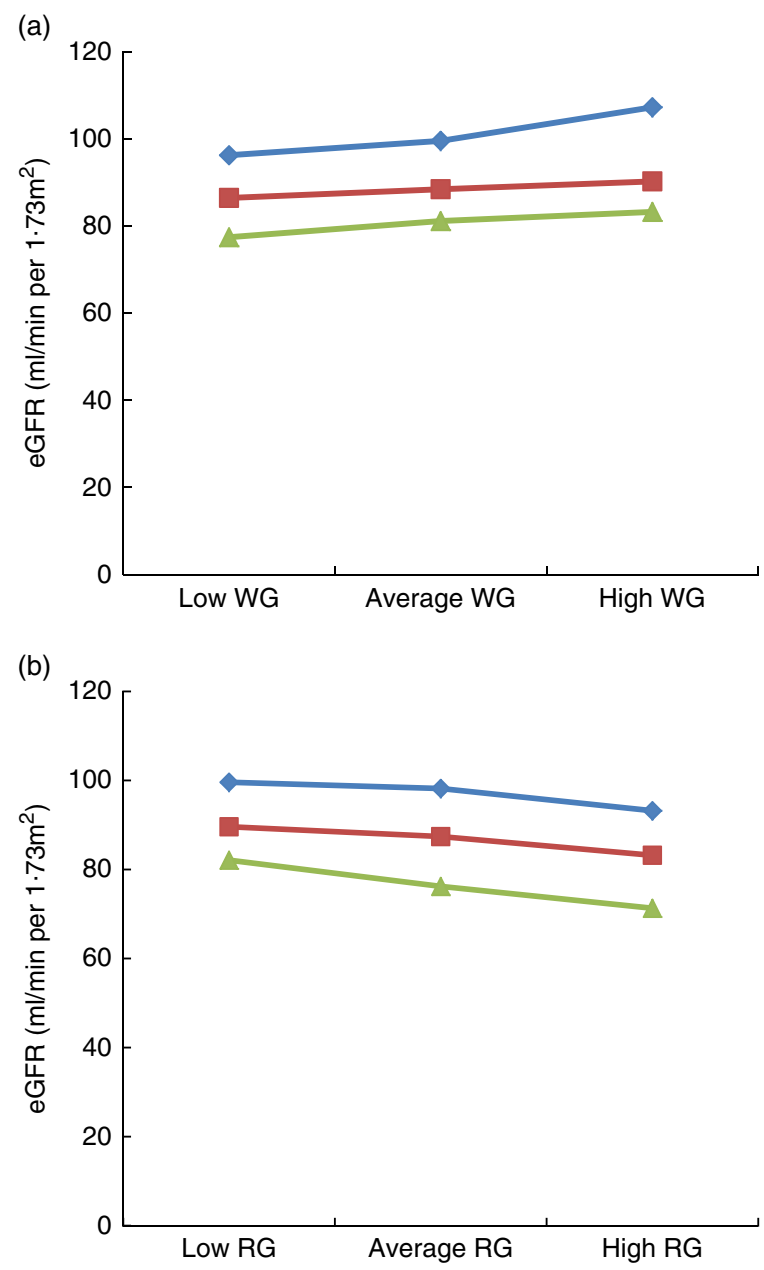

Fig. 1. Impact of BMI on the link between estimated glomerular filtration rate (eGFR), whole grain (WG) and refined grain (RG) consumption. (a) Impact of $\mathrm{BMI}$ on the link between eGFR with WG, (b) impact of BMI on the link between eGFR with RG. Average indicate mean values, low indicates mean -1 SD values and high indicates mean $1 \mathrm{SD}$ values. - , Low $\mathrm{BMI} ;-$, average $\mathrm{BMI}$; $\longrightarrow$, high BMI.
We also found that individuals with a higher intake of WG had lower serum levels of uric acid, while, in contrast, there was a positive association between RG intake and uric acid concentrations. Elevated levels of serum uric acid have been associated with increased cardiovascular risk and $\mathrm{CKD}^{(63-65)}$. In this context, it has been suggested that lowering serum uric acid may improve kidney function ${ }^{(66,67)}$.

The current study has significant clinical and public health implications, regarding the link between WG and RG consumption with renal markers. Our study revealed that, on the one hand, increasing the load of WG (include cereal grains that consist of the intact, or ground, cracked or flaked fruit of the grains) in the diet from half of servings/d to about one-and-ahalf of servings/d could essentially improve the kidney function and chance of CKD, on the other hand, once the intake of the RG increased from one-third of a servings/d to four servings/d it could significantly damage the renal functions and enhance the likelihood of low eGFR. In this regard, a crucial step is necessary towards public health policy making and raising public awareness about the advantages and disadvantages of the WG and RF and choosing the right food basket for the family.

This is the largest study on the association of WG and RG with renal function markers. Participants represented a random sample of the general US population and therefore the results can be extrapolated to the general US population. Because data collection was performed on all weekdays in NHANES, the potential for day-specific information bias is very $\operatorname{low}^{(68,69)}$. Our findings have to be considered along with the context of some study limitations. First, its cross-sectional nature does not allow for direct inference about causality. Second, it is well known that a single $24 \mathrm{~h}$ diet recall interview is not ideal for characterising an individual's long-term habitual intake ${ }^{(70,71)}$. Finally, we did not have data on repeated measurements of exposures and outcomes to evaluate changes over time. So, for future studies it would be suitable to take into consideration some dietary factors that may affect kidney function, 
such as fat intake. We need to mention that although BMI is commonly used to estimate adiposity, this marker might be inaccurate and can lead to bias in measuring adiposity ${ }^{(72)}$. BMI does not take into consideration age, sex, bone structure, fat distribution or muscle mass ${ }^{(72)}$. To overcome this issue, future studies need to use fat mass from bio-impedance which is more accurate than BMI for the adiposity ${ }^{(72)}$. Given the nature of the study, there is always a chance of the residual bias and unmeasured factors that may influence our results, therefore, our results need to be confirmed by randomised controlled trial.

\section{Conclusions}

The results of the present study shed light on the beneficial effects of WG on kidney function and CKD. Furthermore, RG intake was adversely associated with markers of kidney function. These findings suggest the potential utility of modulating diet as a strategy to prevent CKD. The obtained results might have application for both population-wide and high-risk approach to CKD prevention and management in various settings.

\section{Acknowledgements}

This research received no specific grant from any funding agency, or from commercial or not-for-profit sectors.

All authors contributed to: (1) substantial contributions to conception and design, or acquisition of data, or analysis and interpretation of data, (2) drafting the article or revising it critically for important intellectual content, and, (3) final approval of the version to be published.

N. K. has given talks, attended conferences and participated in trials sponsored by Amgen, Angelini, Astra Zeneca, Boehringer Ingelheim, MSD, Novartis, NovoNordisk, Sanofi and WinMedica. D. P. M. has given talks and attended conferences sponsored by MSD, AstraZeneca and Libytec. The other authors have no conflict of interest to declare.

\section{References}

1. Levey AS \& Coresh J (2012) Chronic kidney disease. Lancet 379, $165-180$.

2. Athyros VG, Katsiki N, Karagiannis A, et al. (2012) Editorial: should chronic kidney disease be considered as a coronary heart disease equivalent? Curr Vasc Pharmacol 10, 374-377.

3. Athyros VG, Katsiki N, Karagiannis A, et al. (2012) Stage of chronic kidney disease and severity of coronary heart disease manifestation. Expert Opin Pharmacother 13, 457-460.

4. Honeycutt AA, Segel JE, Zhuo X, et al. (2013) Medical costs of CKD in the Medicare population. J Am Soc Nephrol 24, 1478-1483.

5. Leung KC, Tonelli M \& James MT (2013) Chronic kidney disease following acute kidney injury-risk and outcomes. Nat Rev Nephrol 9, 77-85.

6. Miyamoto T, Carrero JJ \& Stenvinkel P (2011) Inflammation as a risk factor and target for therapy in chronic kidney disease. Curr Opin Nephrol Hypertens 20, 662-668.

7. Giugliano D, Ceriello A \& Esposito K (2006) The effects of diet on inflammation: emphasis on the metabolic syndrome. $J \mathrm{Am}$ Coll Cardiol 48, 677-685.
8. Mazidi M, Kengne AP, Mikhailidis DP, et al. (2017) Dietary food patterns and glucose/insulin homeostasis: a crosssectional study involving 24182 adult Americans. Lipids Health Dis 16, 192.

9. Mazidi M, Kengne AP, Mikhailidis DP, et al. (2018) Effects of selected dietary constituents on high-sensitivity C-reactive protein levels in U.S. adults. Ann Med 50, 1-6.

10. Farhadnejad H, Asghari G, Mirmiran P, et al. (2016) Micronutrient intakes and incidence of chronic kidney disease in adults: Tehran Lipid and Glucose Study. Nutrients $\mathbf{8}, 217$.

11. Huang X, Jiménez-Moleón JJ, Lindholm B, et al. (2013) Mediterranean diet, kidney function, and mortality in men with CKD. Clin J Am Soc Nephrol 8, 1548-1555.

12. Dunkler D, Dehghan M, Teo KK, et al. (2013) Diet and kidney disease in high-risk individuals with type 2 diabetes mellitus. JAMA Intern Med 173, 1682-1692.

13. Huang X, Sjogren P, Arnlov J, et al. (2014) Serum fatty acid patterns, insulin sensitivity and the metabolic syndrome in individuals with chronic kidney disease. I Int Med $\mathbf{2 7 5}$, 71-83.

14. Miller ER 3rd, Juraschek SP, Appel LJ, et al. (2009) The effect of $n$-3 long-chain polyunsaturated fatty acid supplementation on urine protein excretion and kidney function: meta-analysis of clinical trials. Am J Clin Nutr 89, 1937-1945.

15. Xu H, Huang X, Riserus U, et al. (2014) Dietary fiber, kidney function, inflammation, and mortality risk. Clin J Am Soc Nephrol 9, 2104-2110.

16. Lin J, Judd S, Le A, et al. (2010) Associations of dietary fat with albuminuria and kidney dysfunction. Am J Clin Nutr 92, 897-904.

17. Sabatino A, Regolisti G, Gandolfini I, et al. (2017) Diet and enteral nutrition in patients with chronic kidney disease not on dialysis: a review focusing on fat, fiber and protein intake. J Nephrol 30, 743-754.

18. Herber-Gast GM, Boersma M, Verschuren WMM, et al. (2017) Consumption of whole grains, fruit and vegetables is not associated with indices of renal function in the populationbased longitudinal Doetinchem study. Br J Nutr 118, 375-382.

19. Foster MC, Hwang SJ, Massaro JM, et al. (2015) Lifestyle factors and indices of kidney function in the Framingham Heart Study. Am J Nephrol 41, 267-274.

20. Nettleton JA, Steffen LM, Palmas W, et al. (2008) Associations between microalbuminuria and animal foods, plant foods, and dietary patterns in the Multiethnic Study of Atherosclerosis. Am J Clin Nutr 87, 1825-1836.

21. Deniz Ayli M, Ayli M, Ensari C, et al. (2000) Effect of lowprotein diet supplemented with keto acids on progression of disease in patients with chronic renal failure. Nephron $\mathbf{8 4}$, 288-289.

22. Vaziri ND, Said HM, Hollander D, et al. (1985) Impaired intestinal absorption of riboflavin in experimental uremia. Nephron 41, 26-29.

23. Centers for Disease Control and Prevention, National Center for Health Statistics (2019) National Health and Nutrition Examination Survey. http://www.cdc.gov/nchs/nhanes.htm (accessed April 2019).

24. Liu Y (2014) The relationship between lifestyle and self-reported oral health among American adults. Int Dent J 64, 46-51.

25. Needham BL, Adler N, Gregorich S, et al. (2013) Socioeconomic status, health behavior, and leukocyte telomere length in the National Health and Nutrition Examination Survey, 1999-2002. Soc Sci Med (1982) 85, 1-8.

26. Remer T (2001) Influence of nutrition on acid-base balancemetabolic aspects. Eur J Nutr 40, 214-220.

27. Mazidi M, Michos ED \& Banach M (2017) The association of telomere length and serum 25-hydroxyvitamin D levels in US 
adults: the National Health and Nutrition Examination Survey. Arch Med Sci 13, 61-65.

28. Centers for Disease Control and Prevention (2011) Laboratory Procedure Manual. Analyte: C-Reactive Protein. http://www.cdc. gov/NCHS/data/nhanes/nhanes_09_10/CRP_F_met.pdf (accessed August 2013).

29. Selvin E, Manzi J, Stevens LA, et al. (2007) Calibration of serum creatinine in the National Health and Nutrition Examination Surveys (NHANES) 1988-1994, 1999-2004. Am J Kidney Dis 50, 918-926.

30. Chavers BM, Simonson J \& Michael AF (1984) A solid phase fluorescent immunoassay for the measurement of human urinary albumin. Kidney Int 25, 576-578.

31. Grundy SM, Brewer HB Jr, Cleeman JI, et al. (2004) Definition of metabolic syndrome: Report of the National Heart, Lung, and Blood Institute/American Heart Association conference on scientific issues related to definition. Circulation 109, 433-438.

32. Ahluwalia N, Andreeva VA, Kesse-Guyot E, et al. (2013) Dietary patterns, inflammation and the metabolic syndrome. Diabetes Metab 39, 99-110.

33. Ahluwalia N, Dwyer J, Terry A, et al. (2016) Update on NHANES dietary data: focus on collection, release, analytical considerations, and uses to inform public policy. Adv Nutr $\mathbf{7}$, 121-134.

34. Moshfegh AJ, Rhodes DG, Baer DJ, et al. (2008) The US Department of Agriculture automated multiple-pass method reduces bias in the collection of energy intakes. Am J Clin Nutr 88, 324-332.

35. Bowman SAF, Friday JE \& Moshfegh A (2008) MyPyramid Equivalents Database, 2.0 for USDA Survey Foods; Food Surveys Research Group, Beltsville Human Nutrition Research Center, Agricultural Research Service, U.S. Department of Agriculture: Beltsville, MD, USA. http://www.ars.usda.gov/ba/ bhnrc/fsrg (accessed April 2017).

36. Centers for Disease Control and Prevention, National Center for Health Statistics (2005) Analytic and Reporting Guidelines. http://www.cdc.gov/nchs/data/nhanes/nhanes0304/nhane sanalyticguidelinesdec2005.pdf (accessed April 2019).

37. Nwankwo T, Yoon SS, Burt V, et al. (2013) Hypertension among adults in the United States: National Health and Nutrition Examination Survey, 2011-2012. NCHS Data Brief 133, 1-8.

38. Slinker BK \& Glantz SA (1985) Multiple regression for physiological data analysis: the problem of multicollinearity. Am J Physiol 249, R1-R12.

39. Preacher KJ \& Hayes AF (2008) Asymptotic and resampling strategies for assessing and comparing indirect effects in multiple mediator models. Behav Res Methods 40, 879-891.

40. Lin J, Fung TT, Hu FB, et al. (2011) Association of dietary patterns with albuminuria and kidney function decline in older white women: a subgroup analysis from the Nurses' Health Study. Am J Kidney Dis 57, 245-254.

41. Dunkler D, Dehghan M, Teo KK, et al. (2013) Diet and kidney disease in high-risk individuals with type 2 diabetes mellitus. JAMA Int Med 173, 1682-1692.

42. Xu H, Sjögren P, Ärnlöv J, et al. (2015) A proinflammatory diet is associated with systemic inflammation and reduced kidney function in elderly adults. J Nutr 145, 729-735.

43. Khatri M, Moon YP, Scarmeas N, et al. (2014) The association between a Mediterranean-style diet and kidney function in the Northern Manhattan Study cohort. Clin J Am Soc Nephrol 9, $1868-1875$.

44. Ma J, Jacques PF, Hwang SJ, et al. (2016) Dietary guideline adherence index and kidney measures in the Framingham Heart Study. Am J Kidney Dis 68, 703-715.

45. Lin J, Fung TT, Hu FB, et al. (2011) Association of dietary patterns with albuminuria and kidney function decline in older white women: a subgroup analysis from the Nurses' Health Study. Am J Kidney Dis 57, 245-254.

46. Gopinath B, Harris D, Flood V, et al. (2013) A better diet quality is associated with a reduced likelihood of CKD in older adults. Nutr Metab Cardiovasc Dis 23, 937-943

47. Odermatt A (2011) The Western-style diet: a major risk factor for impaired kidney function and chronic kidney disease. $A m J$ Physiol Renal Physiol 301, F919-F931.

48. Nettleton JA, Steffen LM, Mayer-Davis EJ, et al. (2006) Dietary patterns are associated with biochemical markers of inflammation and endothelial activation in the Multi-Ethnic Study of Atherosclerosis (MESA). Am J Clin Nutr 83, 1369-1379.

49. Nerpin E, Helmersson-Karlqvist J, Risérus U, et al. (2012) Inflammation, oxidative stress, glomerular filtration rate, and albuminuria in elderly men: a cross-sectional study. BMC Res Notes $\mathbf{5}, 537$.

50. Qi L, van Dam RM, Liu S, et al. (2006) Whole-grain, bran, and cereal fiber intakes and markers of systemic inflammation in diabetic women. Diabetes Care 29, 207-211.

51. Jensen MK, Koh-Banerjee P, Franz M, et al. (2006) Whole grains, bran, and germ in relation to homocysteine and markers of glycemic control, lipids, and inflammation 1. Am J Clin Nutr 83, 275-283.

52. Lutsey PL, Jacobs DR Jr, Kori S, et al. (2007) Whole grain intake and its cross-sectional association with obesity, insulin resistance, inflammation, diabetes and subclinical CVD: the MESA study. Br J Nutr 98, 397-405.

53. Liese AD, Roach AK, Sparks KC, et al. (2003) Whole-grain intake and insulin sensitivity: the Insulin Resistance Atherosclerosis Study. Am J Clin Nutr 78, 965-971.

54. Steffen LM, Jacobs DR Jr, Murtaugh MA, et al. (2003) Whole grain intake is associated with lower body mass and greater insulin sensitivity among adolescents. Am J Epidemiol 158, 243-250.

55. Athyros VG, Katsiki N, Karagiannis A, et al. (2015) Statins can improve proteinuria and glomerular filtration rate loss in chronic kidney disease patients, further reducing cardiovascular risk. Fact or fiction? Expert Opin Pharmacother 16, 1449-1461.

56. Nikolic D, Banach M, Nikfar S, et al. (2013) A meta-analysis of the role of statins on renal outcomes in patients with chronic kidney disease. Is the duration of therapy important? Int $J$ Cardiol 168, 5437-5447.

57. Nikolic D, Nikfar S, Salari P, et al. (2013) Effects of statins on lipid profile in chronic kidney disease patients: a meta-analysis of randomized controlled trials. Curr Med Res Opin 29, 435-451.

58. Deedwania PC (2014) Statins in chronic kidney disease: cardiovascular risk and kidney function. Postgrad Med 126, 29-36.

59. Marquart L, Slavin JL \& Fulcher TG (editors) (2002) Wholegrain Foods in Health and Disease. St Paul, MN: American Association of Cereal Chemists, Inc.

60. Ajani UA, Ford ES \& Mokdad AH (2004) Dietary fiber and C-reactive protein: findings from national health and nutrition examination survey data. J Nutr 134, 1181-1185.

61. King DE, Egan BM \& Geesey ME (2003) Relation of dietary fat and fiber to elevation of C-reactive protein. Am J Cardiol $\mathbf{9 2}$, $1335-1339$

62. Canfora EE, Jocken JW \& Blaak EE (2015) Short-chain fatty acids in control of body weight and insulin sensitivity. Nat Rev Endocrinol 11, 577-591.

63. Katsiki N \& Mikhailidis DP (2015) Hyperuricaemia in cardiovascular diseases: a passive or an active player? Med Princ Pract 24, 269-270.

64. Katsiki N, Karagiannis A, Athyros VG, et al. (2013) Hyperuricaemia: more than just a cause of gout? J Cardiovasc Med (Hagerstown) 14, 397-402. 
65. Xia X, Luo Q, Li B, et al. (2016) Serum uric acid and mortality in chronic kidney disease: a systematic review and metaanalysis. Metabolism 65, 1326-1341.

66. Mende C (2015) Management of chronic kidney disease: the relationship between serum uric acid and development of nephropathy. Adv Ther 32, 1177-1191.

67. Sampson AL, Singer RF \& Walters GD (2017) Uric acid lowering therapies for preventing or delaying the progression of chronic kidney disease. Cochrane Database Syst Rev 10, Cd009460.

68. Tooze JA, Midthune D, Dodd KW, et al. (2006) A new statistical method for estimating the usual intake of episodically consumed foods with application to their distribution. $J \mathrm{Am}$ Diet Assoc 106, 1575-1587.
69. Guenther PM, Ding EL \& Rimm EB (2013) Alcoholic beverage consumption by adults compared to dietary guidelines: results of the National Health and Nutrition Examination Survey, 2009-2010. J Acad Nutr Diet 113, 546-550.

70. Hebert JR, Hurley TG, Steck SE, et al. (2014) Considering the value of dietary assessment data in informing nutrition-related health policy. Adv Nutr 5, 447-455.

71. Ma Y, Olendzki BC, Pagoto SL, et al. (2009) Number of 24 hour diet recalls needed to estimate energy intake. Ann Epidemiol 19, 553-559.

72. Rothman KJ (2008) BMI-related errors in the measurement of obesity. Int J Obes (2005) 32, Suppl. 3, S56-S59. 